\section{Extreme protection}

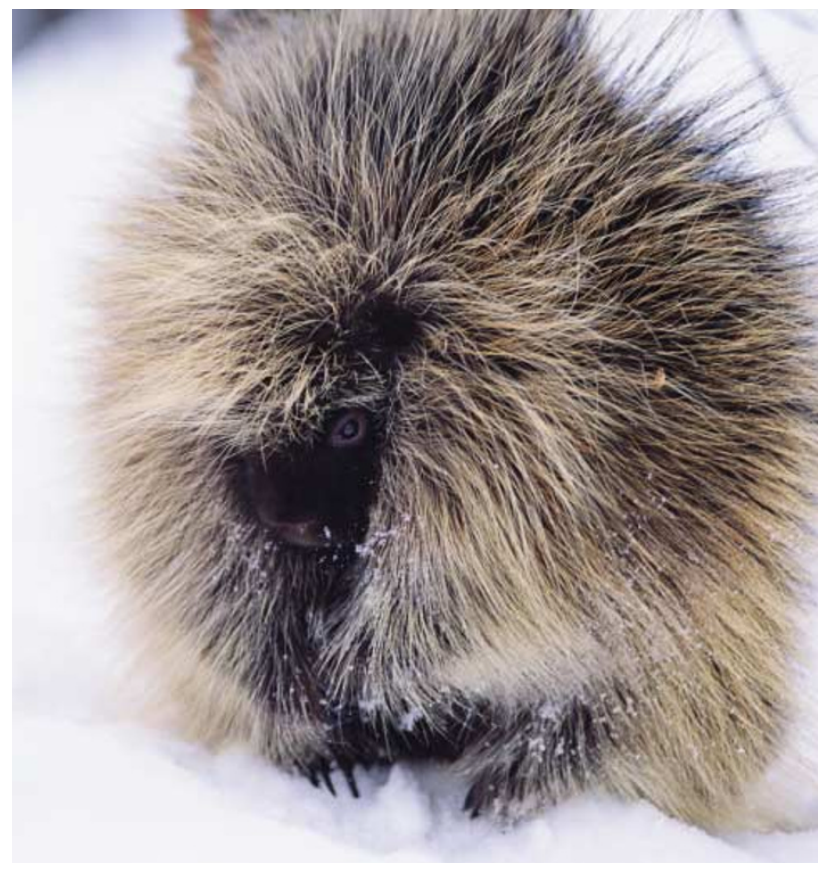

The extremes of linear chromosomes are dangerous locations. Their structural resemblance to double-stranded DNA breaks can trigger unwanted DNA-damage-response pathways with catastrophic results for the cell. Telomeric DNA is therefore enveloped by a 'cap' of protective proteins that conceal these troublesome ends. Now, researchers have shed new light on the function of one of these telomere-binding proteins - TRF2.

TRF2 is a telomeric doublestranded-DNA-binding protein that has a role in the protection of chromosome ends, but its mechanism of action is unknown. A dominant-negative mutation of TRF2 that displaces the protein from its binding site leads to telomeric association of the ATM kinase - a critical mediator of the DNA-damage-response pathway. These findings indicate that TRF2 might oppose the action or the recruitment of ATM.

To investigate this further, Titia de Lange and co-workers overexpressed TRF2 in human primary fibroblasts, exposed these cells to ionizing radiation (IR) and then analysed the ATM-mediated response to DNA damage. Microscopy analysis revealed that an increased percentage of these cells had entered mitosis - indicating a failure of ATM-mediated cell-cycle arrest. Quantitative immunoblots showed decreased levels of the p53 protein and its downstream targets, and ATM-dependent phosphorylation of NBS1 - a DNA-doublestrand-break-repair protein - was also impaired in these cells.

DNA damage that is induced by low levels of IR is usually associated with activation of ATM through autophosphorylation. However, when the authors co-expressed TRF2 and ATM, the level of phosphorylated ATM after IR exposure was reduced compared with controls. Similarly, endogenous ATM phosphorylation was reduced in cells that overexpressed TRF2.

So, does TRF2 directly interfere with ATM activation and function? Anti-ATM antibody co-precipitated

\section{EPIGENETICS}

\section{Silent transmission}

\author{
In mammals, DNA and histone methylation \\ together provide an effective, long-term \\ mechanism for silencing gene expression, but \\ how specific methylation patterns are \\ 'remembered' during cell division is unclear. \\ In a recent paper, Sarraf and Stancheva \\ showed that this depends on the coupling of \\ the two types of methylation during DNA \\ replication. \\ At sites of constitutive heterochromatin \\ and transcriptionally silenced promoters, \\ silencing is mediated by methylation of \\ DNA at $\mathrm{CpG}$ dinucleotides and of \\ histone $\mathrm{H} 3$ at lysine 9 (H3-K9). During \\ DNA replication, the methyltransferase \\ DNMT1 interacts with the replication \\ machinery to ensure that DNA methylation \\ patterns are faithfully copied. By contrast, \\ little is known about how histone \\ methylation is reproduced. One model \\ proposes that this is somehow coordinated \\ with DNA methylation, but evidence has so \\ far been lacking.
}

By co-immunoprecipitation, Sarraf and Stancheva showed that MBD1 - a protein that specifically binds methyl-CpG groups associates with a complex that contains an H3-K9-specific methyltransferase activity, providing a possible link between DNA and histone methylation. The other components of the complex were identified as the $\mathrm{H} 3-\mathrm{K} 9-$ specific methyltransferase SETDB1 and CAF1, a protein involved in chromatin assembly. So, MBD1 bound to methylated DNA could recruit SETDB1 and, through its interaction with $\mathrm{CAF} 1$, promote $\mathrm{H} 3-\mathrm{K} 9$ methylation at specific sites during chromatin assembly.

Consistent with this, the three proteins were shown to form a complex in vivo specifically during DNA replication. The authors also showed how DNA replication is coupled to the activation of the CAF1-MBD1-SETDB1 complex. CAF1 is only transiently associated with MBD1 and SETDB1 during S-phase and this depends on MBD1 being displaced from DNA. Specific inhibition of replication elongation showed that this displacement depends on the progression of the replication complex. This seems to knock MBD1 off the DNA strand, allowing it to bind $\mathrm{CAF} 1$ and promote $\mathrm{H} 3-\mathrm{K} 9$ methylation on newly formed chromatin.

How does this relate to the silencing of specific genes? Sarraf and Stancheva identified several genomic MBD1-binding sites, including a $\mathrm{CpG}$ island in the $p 53 B P 2$ promoter region. In HeLa cells, $p 53 B P 2$ is usually transcriptionally silent, and the authors showed that this depends on both DNA methylation and methylation at $\mathrm{H} 3-\mathrm{K}$. Treatment with an inhibitor of DNA methylation or with small interfering RNAs against either MBD1 or SETDB1 led to loss of methylated $\mathrm{H} 3-\mathrm{K} 9$ at the $p 53 B P 2$ promoter and induced the expression of the gene. Similarly, reducing levels of DNA methylation or MBD1 expression resulted in loss of methylated $\mathrm{H} 3-\mathrm{K} 9$ at 23 other genomic MBD1-binding sites.

So, interactions between the DNA and histone methylation machinery seem to be important for maintaining patterns of epigenetic modification on a widespread basis. This provides one way of ensuring that transcriptional silencing is transmitted accurately through ongoing rounds of cell division, an essential requirement for normal mammalian development.

Louisa Flintoft

Assistant Editor, Nature Reviews

\section{(2) References and links}

\section{ORIGINAL RESEARCH PAPER}

Sarraf, S. A. \& Stancheva, I. Methyl-CpG binding protein MBD1 couples histone $H 3$ methylation at lysine 9 by SETDB1 to DNA replication and chromatin assembly. Mol. Cell 15, 595-605 (2004)

\section{WEB SITE}

Irina Stancheva's laboratory: http://www.bms.ed. ac.uk/services/staff/stancheva/index.htm 